\title{
Effects of Disopyramide and Mexiletine on the Terminal Repolarization Process of the In Situ Heart Assessed Using the Halothane-Anesthetized In Vivo Canine Model
}

\author{
Hiroshi Yoshida, MD*; Atsushi Sugiyama, MD; Yoshioki Satoh, MD; \\ Yuko Ishida, PhD; Kiyotaka Kugiyama, MD*; Keitaro Hashimoto, MD
}

\begin{abstract}
This study was designed to assess the effects of typical class I drugs on the terminal repolarization process of the in situ heart, which is a useful marker of the potential of drug-induced long QT syndrome. Disopyramide (0.3 and $3.0 \mathrm{mg} / \mathrm{kg}$ per $10 \mathrm{~min}, \mathrm{n}=6)$ or mexiletine $(0.3$ and $3.0 \mathrm{mg} / \mathrm{kg}$ per $30 \mathrm{~s}, \mathrm{n}=6)$ was intravenously administered to halothane-anesthetized beagle dogs under the monitoring of multiple cardiovascular parameters. Antiarrhythmic concentrations were obtained with the high dose of each drug. The low dose of disopyramide or mexiletine hardly affected any of the electrophysiological parameters assessed. The high dose of disopyramide prolonged the monophasic action potential duration (MAP90) and effective refractory period (ERP) to a similar extent, thus displacing the terminal repolarization period backward, which might provide a potential proarrhythmic substrate, particularly at a slow heart rate. On the other hand, the high dose of mexiletine shortened the MAP90, but prolonged the ERP, resulting in the disappearance of the terminal repolarization period, which could prevent premature excitation with its associated conduction slowing. These electrophysiological effects of disopyramide and mexiletine on the terminal repolarization phase may at least in part explain their clinically described antiarrhythmic and proarrhythmic properties. (Circ J 2002; 66: 857-862)
\end{abstract}

Key Words: Disopyramide; Long QT syndrome; Mexiletine; Terminal repolarization process

D rug-induced long QT syndrome is currently a hot topic of concern for the pharmaceutical companies as well as for clinicians!,2 We have previusly assessed the effects of class II, III and IV antiarrhythmic agents and some non-cardiovascular drugs on the repolarization process using the halothane-anesthetized canine in vivo model, and found that the effects of the drugs on phase 3 repolarization in this model are quite useful for predicting drug-induced QT prolongation that might trigger torsades de pointes in clinical practice?-11

Class I drugs are known to affect the QT interval, ${ }^{12,13}$ but information regarding the effects of class I antiarrhythmic drugs in this type of model is still limited $!^{14-17}$ In this study, we simultaneously assessed the in vivo electrophysiological and cardiohemodynamic effects of typical class I antiarrhythmic drugs, disopyramide and mexiletine, using the halothane-anesthetized canine model. To better analyze the electrophysiological effects on the depolarization/repolarization process, we recorded His bundle electrograms and monophasic action potentials (MAPs), respectively, in addition to the standard lead II surface ECG. Moreover, a MAP recording/pacing combination catheter was used to

(Received April 19, 2002; revised manuscript received June 5, 2002; accepted June 11, 2002)

Department of Pharmacology and *Second Department of Internal Medicine, Yamanashi Medical University, Tamaho-cho, Nakakomagun, Yamanashi, Japan

Mailing address: Atsushi Sugiyama, MD, Department of Pharmacology, Yamanashi Medical University, Tamaho-cho, Nakakoma-gun, Yamanashi 409-3898, Japan. E-mail: atsushis@ res.yamanashi-med. ac.jp simultaneously measure both MAP and effective refractory period (ERP) at the same site to assess the drug effects on the terminal repolarization period (phase 3 ) of the action potential? ${ }^{-11,14-17}$

\section{Methods}

All experiments were performed in accordance with the Guidelines for Animal Experiments, Yamanashi Medical University. Experiments were carried out using beagle dogs weighing approximately $10 \mathrm{~kg}$. Animals were obtained through the Animal Laboratory for Research of Yamanashi Medical University. Dogs were anesthetized initially with thiopental sodium $(30 \mathrm{mg} / \mathrm{kg}$, iv) and after intubation with a cuffed endotracheal tube, $1.0 \%$ halothane vaporized with $100 \%$ oxygen was inhaled with a volume-limited ventilator (SN-480-3; Shinano, Tokyo, Japan). Tidal volume and respiratory rate were set at $20 \mathrm{ml} / \mathrm{kg}$ and 15 stroke/min, respectively. To prevent blood clotting, heparin calcium (100 units/kg, iv) was administered.

Cardiohemodynamic and Electrophysiological Parameters

The surface lead II ECG was obtained from the limb electrodes and the corrected QT interval (QTc) was calculated using Bazett's formula: ${ }^{18}$ The systemic blood pressure (BP) was measured at the left femoral artery, and a pig-tail catheter was positioned at the left ventricle through the left femoral artery to measure the left ventricular pressure. The maximum upstroke velocity of the left ventricular pressure $(\mathrm{LVdP} / \mathrm{dtmax})$ and the left ventricular end-diastolic pressure (LVEDP) were obtained to estimate the contractility and 

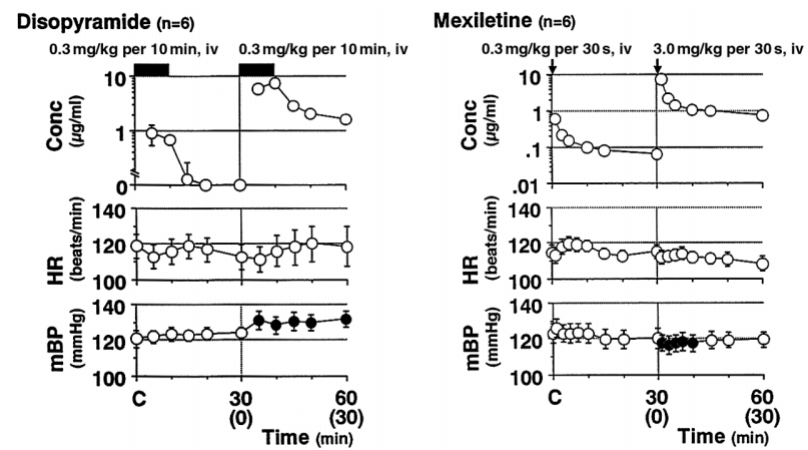

Fig 1. Time courses of the plasma drug concentration (Conc), heart rate $(\mathrm{HR})$ and mean blood pressure $(\mathrm{mBP})$ after the administration of disopyramide (left panels) and mexiletine (right panels). Data are presented as the mean \pm SE. The closed symbols represent the significant differences from each control value (C) by $\mathrm{p}<0.05$.

preload of the left ventricle, respectively. A quad-polar electrodes catheter was positioned at the non-coronary cusp through the right femoral artery to obtain the His bundle electrogram. A thermodilution catheter (TC-704; Nihon Kohden, Tokyo, Japan) was positioned at the right side of the heart through the right femoral vein and the cardiac output $(\mathrm{CO})$ was measured by a standard thermodilution method using a CO computer (MCF-1100, Nihon Kohden). Total peripheral vascular resistance (TPR) was calculated using the basic equation: $\mathrm{TPR}=$ mean $\mathrm{BP} / \mathrm{CO}$.

A bi-directional steerable MAP recording/pacing combination catheter (1675P; EP Technologies Inc, Sunnyvale, CA, USA) was positioned on the endocardium of the right ventricle through the left femoral vein. The MAP signals were amplified with a DC preamplifier (model 300, EP Technologies Inc) and the amplitude of the MAP was measured as the distance from the diastolic baseline to the crest of the MAP plateau phase as reported previously 16 The duration of the MAP signal was measured as an interval, along a line horizontal to the diastolic baseline, from the MAP upstroke to the desired repolarization level. The interval at $90 \%$ repolarization was defined as MAP90.

The heart was electrically driven via the pacing electrodes of the combination catheter using a cardiac stimulator (SEC-3102, Nihon Kohden). The stimulation pulses were rectangular in shape, $1-2 \mathrm{~V}$ of amplitude (approx. twice the threshold voltage) and of $1 \mathrm{~ms}$ duration. The MAP 90 was measured during the sinus rhythm (MAP90(sinus)) and at pacing cycle lengths of $400 \mathrm{~ms}$ (MAP90(CL400)) and $300 \mathrm{~ms}$ (MAP90(CL300)). In addition, the ERP of the ventricle was assessed by programmed electrical stimulation via the pacing electrodes of the combination catheter. The pacing protocol consisted of 5 beats of basal stimuli in a cycle length of $400 \mathrm{~ms}$ followed by an extrastimulus of various coupling intervals. Starting in late diastole, the coupling interval was shortened in $5-10 \mathrm{~ms}$ decrements until refractoriness occurred. The terminal repolarization period (TRP) of the action potential was calculated as TRP = MAP90(CL400)-ERP, which would reflect the extent of the electrical vulnerability of the heart? ${ }^{3} 11,14-17,19$

\section{Experimental Protocol}

The animals were randomized into 2 groups; namely, disopyramide-administered group $(n=6)$ and mexiletineadministered group $(n=6)$. The systemic BP, left ventricular pressure, ECG, His bundle electrogram and MAP
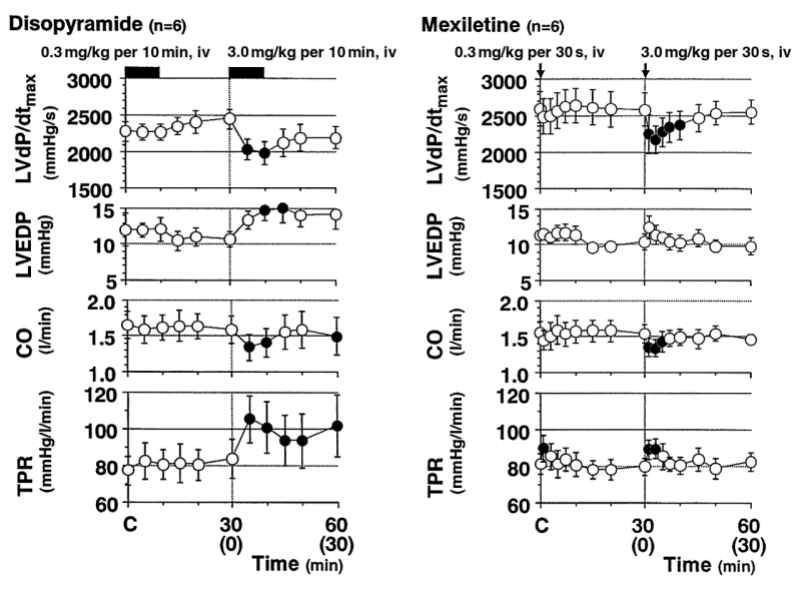

Fig 2. Time courses of the maximum upstroke velocity of the left ventricular pressure ( $\mathrm{LVdP} / \mathrm{dtmax}$ ), left ventricular end-diastolic pressure (LVEDP), cardiac output (CO) and total peripheral resistance (TPR) after the administration of disopyramide (left panels) and mexiletine (right panels). Data are presented as the mean \pm SE. The closed symbols represent the significant differences from each control value $(\mathrm{C})$ by $\mathrm{p}<0.05$.

signals were continuously monitored using a polygraph system (RM-6000, Nihon Kohden), and analyzed using a real time fully automatic data analysis system (MP/VAS 3 for Macintosh V.1.0; Physio-Tech, Tokyo, Japan). The cardiovascular variables were assessed in the following order at each time point. CO was measured twice; the ECG, His bundle electrogram, systemic BP and left ventricular pressure and MAP signal were analyzed during sinus rhythm; then the MAP signals were analyzed during ventricular pacing at the cycle lengths of 400 and $300 \mathrm{~ms}$; finally, the ERP of the ventricle was measured. All data were usually obtained within $1 \mathrm{~min}$ at each time point.

In the disopyramide-administered group, after each basal control cardiovascular value was assessed, a low dose of $0.3 \mathrm{mg} / \mathrm{kg}$ was intravenously administered over $10 \mathrm{~min}$ and the effects of the drug on each cardiovascular parameter were assessed at 5,10,15, 20 and $30 \mathrm{~min}$ after the start of infusion. Disopyramide at a high dose of $3.0 \mathrm{mg} / \mathrm{kg}$, which has been demonstrated to exert an antiarrhythmic effect in canine ventricular arrhythmia models ${ }^{20}$ was then administered over $10 \mathrm{~min}$ and the effect on each parameter was assessed in the same manner.

In the mexiletine-administered group, after each basal control value was assessed, a low dose of $0.3 \mathrm{mg} / \mathrm{kg}$ was intravenously administered over $30 \mathrm{~s}$ and the effects of the drug on each cardiovascular parameter were assessed at 1 , $3,5,7,10,15,20$ and $30 \mathrm{~min}$ after the start of injection. Mexiletine in a high dose of $3.0 \mathrm{mg} / \mathrm{kg}$, which also has an antiarrhythmic effect ${ }^{20}$ was subsequently administered over $30 \mathrm{~s}$ and its effect on each parameter assessed in the same manner.

A volume of $3 \mathrm{ml}$ of blood was drawn from the right femoral artery at each time point to measure the plasma drug concentration. The blood samples were centrifuged at $1,500 \mathrm{G}$ for $30 \mathrm{~min}$ at $4^{\circ} \mathrm{C}$ and the plasma was stored at $-80^{\circ} \mathrm{C}$ until the drug concentration was measured. Sensitive and specific determinations of the concentrations of disopyramide and mexiletine were performed at SRL Co, Ltd (Tokyo, Japan) using a standard high-performance liquid chromatographic method. The limit of quantification of disopyramide and mexiletine was $0.2 \mu \mathrm{g} / \mathrm{ml}$ and $0.02 \mu \mathrm{g} / \mathrm{ml}$, 


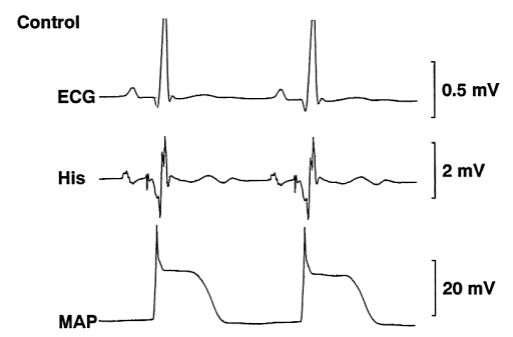

$10 \mathrm{~min}$ after $3.0 \mathrm{mg} / \mathrm{kg}$ per $10 \mathrm{~min}$, iv Disopyramide

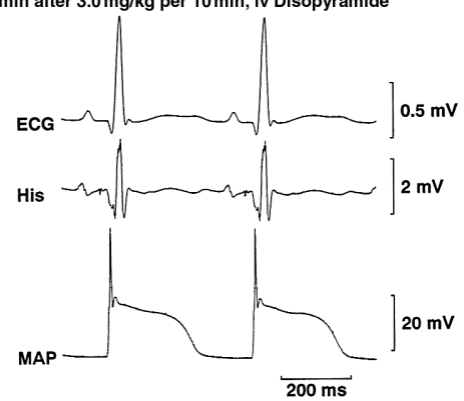

Fig 3. Typical tracings of lead II surface ECG, His bundle electrogram (His) and monophasic action potentials (MAP) recorded from the right ventricle during sinus rhythm before (Control) and $10 \mathrm{~min}$ after the administration of disopyramide. Prolongation of both QT interval and MAP duration were observed after the administration of disopyramide.

respectively.

\section{Drugs}

The following drugs were purchased: disopyramide phosphate (Chugai, Tokyo, Japan), mexiletine hydrochloride (Boehringer, Hyogo, Japan), thiopental sodium (Tanabe, Osaka, Japan), halothane (Takeda, Tokyo, Japan) and heparin calcium (Mitsui, Tokyo, Japan).

\section{Statistics}

Data are presented as the mean $\pm \mathrm{SE}$. The statistical difference within a parameter was evaluated by paired ttest or one-way, repeated-measures analysis of variance (ANOVA) followed by Contrasts for mean values comparison, while that of unpaired data between the groups was evaluated by unpaired t-test. A p value of less than 0.05 was considered significant.

\section{Results}

There was no statistically significant difference in each pre-drug control value between the disopyramide- and mexiletine-administered groups except for QTc. Neither spontaneously occurring ventricular premature contraction nor cardiovascular collapse was observed in any dog during the whole experimental period.

\section{Plasma Drug Concentrations}

The time courses of the plasma concentrations of disopyramide $(n=6)$ and mexiletine $(n=6)$ are summarized in Fig 1. The peak plasma drug concentrations after 0.3 and $3.0 \mathrm{mg} / \mathrm{kg}$ of disopyramide administration were $0.90 \pm 0.37$ and $7.65 \pm 0.61 \mu \mathrm{g} / \mathrm{ml}$, respectively, and $0.59 \pm 0.10$ and $7.37 \pm 037 \mu \mathrm{g} / \mathrm{ml}$, respectively, for 0.3 and $3.0 \mathrm{mg} / \mathrm{kg}$ of mexiletine. The decrease in the plasma concentration of both drugs followed a pattern that could be predicted by the
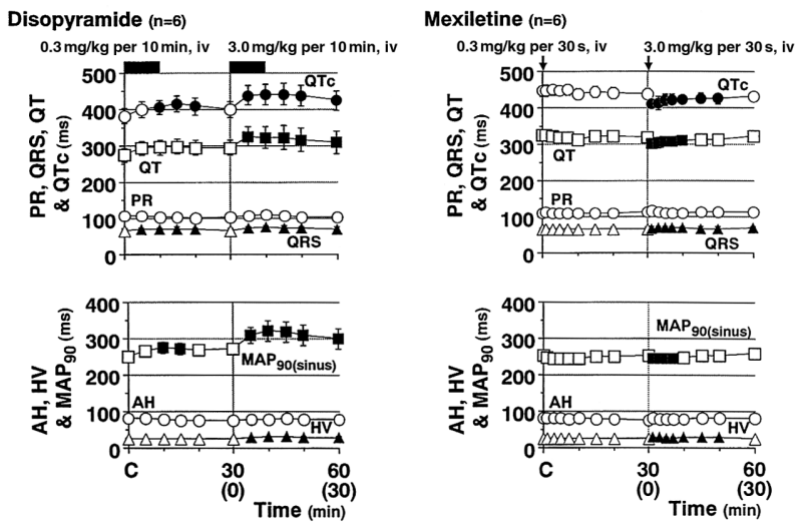

Fig 4. Time courses of PR interval (circles), QRS width (triangles), QT interval (squares) and QTc (circles) (upper panels); and those of AH interval (circles), HV interval (triangles) and MAP90(sinus) (squares) (lower panels) after the administration of disopyramide (left panels) and mexiletine (right panels). All of these parameters were recorded during sinus rhythm. Data are presented as the mean \pm SE. The closed symbols represent the significant differences from each control value $(\mathrm{C})$ by $\mathrm{p}<0.05$. AH, atrio-His; HV, His-ventricular; MAP 90 , monophasic action potential duration at $90 \%$ recovery level; MAP90(sinus), MAP90 during sinus rhythm.

two-component theory of pharmacokinetics.

\section{Effects on the HR and Mean BP}

The time courses of the HR and mean BP are summarized in Fig 1. At the pre-drug control, the HR (beats/min) and mean BP $(\mathrm{mmHg})$ were $119 \pm 7$ and $121 \pm 5$ in the disopyramide group $(n=6)$, and $114 \pm 5$ and $123 \pm 5$ in the mexiletine group $(\mathrm{n}=6)$, respectively. In the disopyramide group, no significant change was observed in HR, but the mean BP increased, and significant changes were detected for 5-30 min after the high dose. In the mexiletine group, no significant change was observed in $\mathrm{HR}$, but the mean BP decreased and significant changes were detected for 1-10 min after the high dose.

\section{Effects on the LVdP/dtmax and LVEDP}

The time courses of the LVdP/dtmax and LVEDP are summarized in Fig 2. The $\mathrm{LVdP} / \mathrm{dtmax}(\mathrm{mmHg} / \mathrm{s})$ and LVEDP ( $\mathrm{mmHg}$ ) at the pre-drug control were, respectively, $2,275 \pm 133$ and $12.0 \pm 2.3$ in the disopyramide group $(n=6)$, and 2,594 \pm 240 and $11.4 \pm 0.6$ in the mexiletine group ( $n=6)$. In the disopyramide group, the LVdP/dtmax decreased and significant changes were detected for 5-10 min after the high dose; the LVEDP increased and significant changes were detected for $10-15 \mathrm{~min}$ after the high dose. In the mexiletine group, the LVdP/dtmax also decreased and significant changes were detected for 1-10 min after the high dose administration, but no significant change was observed in the LVEDP.

\section{Effects on the CO and TPR}

The time courses of the $\mathrm{CO}$ and TPR are summarized in Fig 2. The $\mathrm{CO}(\mathrm{L} / \mathrm{min})$ and TPR $(\mathrm{mmHg} \cdot \mathrm{min} / \mathrm{L})$ at the pre-drug control were, respectively, $1.65 \pm 0.19$ and $77 \pm 8$ in the disopyramide group $(\mathrm{n}=6)$, and $1.56 \pm 0.15$ and $81 \pm 6$ in the mexiletine group $(n=6)$. In the disopyramide group, the $\mathrm{CO}$ decreased and significant changes were detected for 5-10 min and at $30 \mathrm{~min}$ after the high dose; the TPR increased and significant changes were detected for 

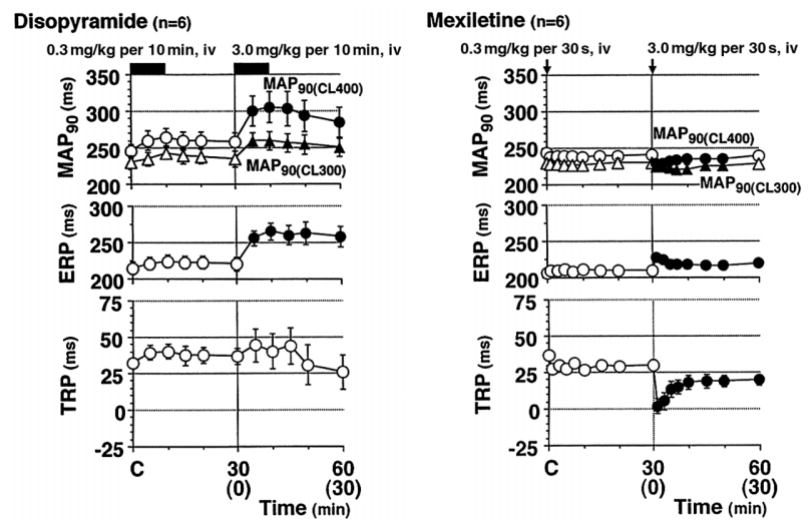

Fig 5. Time courses of MAP90(CL300) and MAP90(CL400), ERP and TRP after the administration of disopyramide (left panels) or mexiletine (right panels). Peak values (ms) of ERP and TRP were, respectively, $265 \pm 12$ and $44 \pm 12$ in the disopyramide group, and $227 \pm 6$ and $2 \pm 5$ in the mexiletine group. Data are presented as the mean \pm SE. The closed symbols represent the significant differences from each control value (C) by $\mathrm{p}<0.05$. MAP90(CL300) and MAP90(CL400), MAP90 during the electrical pacing at a cycle length of $300 \mathrm{~ms}$ and $400 \mathrm{~ms}$; ERP, effective refractory period at a basic cycle length of $400 \mathrm{~ms}$; TRP, terminal repolarization period $(\mathrm{TRP}=\mathrm{MAP} 90(\mathrm{CL} 400)-\mathrm{ERP})$.

5-30 min after the high dose. In the mexiletine group, the $\mathrm{CO}$ also decreased and significant changes were detected for 1-5 min after the high dose; the TPR also increased and significant changes were detected at 1 min after the low dose and for 1-3 min after the high dose.

\section{Effects on the ECG During Sinus Rhythm}

Typical ECG traces after the administration of disopyramide and mexiletine are shown in Fig 3, and the time courses of the parameters of ECG are summarized in Fig 4. The PR interval, QRS width, QT interval and QTc (ms) at the pre-drug control were, respectively, 106 $\pm 6,66 \pm 5$, $274 \pm 18$ and $383 \pm 21$ in the disopyramide group $(n=6)$, and $110 \pm 3,64 \pm 5,324 \pm 13$ and $446 \pm 14$ in the mexiletine group $(n=6)$. In the disopyramide group, no significant change was observed in the PR interval, but the QRS width, QT interval and QTc were prolonged. Significant prolongation was observed in (1) the QRS width for 5-20 min after the low dose and for 5-30 min after the high dose, (2) the QT interval for 5-30 min after the high dose and (3) the QTc for $10-20 \mathrm{~min}$ after the low dose and for 5-30 min after the high dose. In the mexiletine group, no significant change was observed in the PR interval, but the QRS width was prolonged and both the QT interval and QTc were shortened. Significant prolongation was observed in the QRS width for 1-30 min after the high dose and significant shortening was observed in the QT interval for $1-10 \mathrm{~min}$ and in the QTc for 1-20 min after the same dose.

\section{Effects on the AH and HV Intervals and MAP9o During Sinus Rhythm}

Typical His bundle electrogram traces and MAP during sinus rhythm are shown in Fig 3, and the time courses of the atrio-His ( $\mathrm{AH})$ and His-ventricular (HV) intervals and MAP90(sinus) are summarized in Fig 4. The pre-drug control values (ms) were, respectively, $80 \pm 7,25 \pm 1$ and $249 \pm 12$ in the disopyramide group $(n=6)$, and $82 \pm 3,25 \pm 1$ and $253 \pm 4$ in the mexiletine group $(n=6)$. In the disopyramide group, no significant change was observed in the $\mathrm{AH}$ interval during the experimental period, but the HV interval and MAP90(sinus) were prolonged. Significant prolongation was observed in the HV interval for 5-30 min after the high dose, and in the MAP90(sinus) for 10-15 min after the low dose and for 5-30 min after the high dose. The peak prolongation of MAP90(sinus) was observed at 10 min after the high dose and was $322 \pm 28 \mathrm{~ms}$. In the mexiletine group, no significant change was observed in the AH interval, but the $\mathrm{HV}$ interval was prolonged and the MAP90(sinus) was shortened. Significant prolongation was observed in the HV interval for 1-20 min and as well as significant shortening in the MAP90(sinus) for $1-7 \mathrm{~min}$ after the high dose.

\section{Effects on the MAP90(CL400), MAP90(CL300), ERP and TRP During Ventricular Pacing}

The time courses of the MAP90(CL400), MAP90(CL300), ERP and TRP are summarized in Fig 5. The pre-drug control values (ms) were, respectively, 246 $\pm 9,230 \pm 9$, $214 \pm 9$ and $32 \pm 2$ in the disopyramide group $(n=6)$, and $243 \pm 3,230 \pm 4,207 \pm 4$ and $37 \pm 3$ in the mexiletine group $(\mathrm{n}=6)$. In the disopyramide group, the MAP90(CL400), MAP90(CL300) and ERP were prolonged, but no significant change was observed in the TRP. Significant prolongation in MAP90(CL400), MAP90(CL300) and ERP was observed for 5-30 min after the high dose and the peak prolongation of MAP90(CL400) occurred at $10 \mathrm{~min}$ after the high dose at $305 \pm 21 \mathrm{~ms}$. No significant difference was detected between the peak values for MAP90(sinus) and MAP90(CL400). In the mexiletine group, MAP90(CL400), MAP90(CL300) and TRP were shortened, and the ERP was prolonged. Significant shortening in the MAP90(CL400) and MAP90(CL300) was observed for 1-20 min after the high dose, and significant change in the ERP and TRP was detected for 1-30 min after the high dose. In addition, the time courses of the absolute change in MAP90(CL400) and MAP90(CL300) from their respective pre-drug control values were calculated (data not shown) to examine the reverse frequency-dependent change of the repolarization process. In the disopyramide group, the increment tended to be greater in the MAP90(CL400) than in the MAP90(CL300), but did not achieve the statistical significance. Meanwhile, in the mexiletine group, no significant difference was detected between the decrements in the MAP90(CL400) and MAP90(CL300).

\section{Discussion}

The present study used the halothane-anesthetized canine model to simultaneously assess the acute in vivo electrophysiological and cardiohemodynamic actions of typical class I antiarrhythmic drugs, disopyramide and mexiletine, to better understand their clinical utility and adverse effects? ${ }^{3-11,14}$

\section{Plasma Drug Concentrations}

In our previous studies using digitalis-, epinephrine- and coronary ligation-induced canine ventricular arrhythmia models, the minimum effective plasma concentrations of disopyramide and mexiletine were $1.7-4.2 \mu \mathrm{g} / \mathrm{ml}$ and $1.8-$ $3.7 \mu \mathrm{g} / \mathrm{ml}$, respectively. ${ }^{20}$ In addition, previous clinical studies have shown that therapeutic drug concentrations of disopyramide and mexiletine are $2-5 \mu \mathrm{g} / \mathrm{ml}$ and $0.7-$ $1.6 \mu \mathrm{g} / \mathrm{ml}$, respectively ${ }^{12,13}$ Thus, the plasma drug concentrations achieved in the present study indicate that experimental and clinical antiarrhythmic concentrations of both drugs can be obtained by intravenous administration 
of $3.0 \mathrm{mg} / \mathrm{kg}$ in this canine model.

\section{Cardiohemodynamic Effects}

The high dose of disopyramide increased the meanBP and preload to the left ventricle, suppressed ventricular contraction, but hardly affected sinus automaticity. In contrast, the high dose of mexiletine slightly decreased the mean BP, suppressed ventricular contraction, but hardly affected either the preload to the left ventricle or sinus automaticity. These cardiohemodynamic effects were essentially in accordance with previous reports ${ }^{12-14,21}$ More importantly, it should be noted that mexiletine as well as disopyramide suppressed ventricular contraction, because the negative inotropic effect of class $\mathrm{Ib}$ antiarrhythmic drugs, such as lidocaine, is commonly thought to be clinically insignificant ${ }^{13}$

\section{In Vivo Electrophysiological Effects}

Disopyramide and mexiletine both inhibited intraventricular conduction, which depends solely on the sodium current ${ }^{2}$ confirming their well-known inhibitory effect on the sodium channels ${ }^{23,24}$ On the other hand, disopyramide prolonged the ventricular repolarization phase during ventricular pacing as well as during sinus rhythm, whereas mexiletine shortened it. Disopyramide has been reported to inhibit several potassium currents, including IKr (rapidly activating component of delayed rectifier $\mathrm{K}^{+}$current), Ito (transient outward $\mathrm{K}^{+}$current), IK1 (inward rectifier $\mathrm{K}^{+}$ current) IKACh (muscarinic acetylcholine receptor-operated $\mathrm{K}^{+}$current) and IKaTP (ATP-sensitive $\mathrm{K}^{+}$current)? ${ }^{25-28} \mathrm{Al}-$ though we could not detect significant differences between the disopyramide-induced increments in MAP90(CL400) and MAP90(CL300), it has been reported that disopyramide may exert a reverse frequency-dependent prolongation of the repolarization period at a slow $\mathrm{HR}^{29}$ On the other hand, mexiletine is known to reduce the sodium window current and calcium current in addition to activating the IKATP currents ${ }^{30,31}$ Thus, the previous knowledge from the in vitro studies may at least in part explain the currently observed in vivo effects of the drugs on the ventricular repolarization phase. Moreover, the in vivo canine model can be considered to bridge the gap between basic research reports and the clinically observed electrophysiological profile of disopyramide and mexiletine!2,13,25-31

\section{Effects on the Terminal Repolarization Phase}

In our recent study using the canine isolated, bloodperfused ventricular tissue preparation, ${ }^{19}$ we demonstrated that prolongation and backward shift of the TRP enhanced the chance of conduction slowing at a less complete repolarization level, which is associated with a high incidence of torsades de pointes. In the present study, disopyramide prolonged the MAP90 and ERP to a similar extent at a HR of 150 beats/min, thus displacing the TRP backward in the cardiac cycle, potentially increasing the likelihood of the $\mathrm{R}$ on $\mathrm{T}$ phenomenon. As the effects of disopyramide on the ERP might be attenuated and those on the MAP90 enhanced at a slower $\mathrm{HR}^{29}$ one can speculate that disopyramide might prolong the TRP during bradycardia, and that the reverse would be true in the case of tachyarrhythmia. Experiments are now on-going to demonstrate that hypothesis using canine complete atrioventricular conduction block models in which HR can be controlled by ventricular pacing over $40-200$ beats $/ \mathrm{min}^{32}$ On the other hand, mexiletine shortened the MAP90, but prolonged the ERP, resulting in the disappearance of TRP, which could prevent premature excitation with its associated conduction slowing 14 These differences in the electrophysiological profiles of these drugs might explain their clinically described antiarrhythmic and proarrhythmic effects! $1,2,12,13$

\section{Conclusions}

Antiarrhythmic concentrations of mexiletine as well as disopyramide exert a negative inotropic effect. As well as their well-known electrophysiological properties, disopyramide displaces the TRP backward in the cardiac cycle, whereas mexiletine significantly shortens it, which could be their antiarrhythmic and proarrhythmic mechanisms. Thus, the currently used in vivo canine model is useful for better understanding the relationship between the basic electrophysiological observations in vitro and the clinical experience of these drugs.

\section{Acknowledgments}

The authors thank Mr Y. Nakamura and Mrs A. Moriya for their skillful technical assistance.

\section{References}

1. Tamargo J. Drug-induced torsade de pointes: From molecular biology to bedside. Jpn J Phamacol 2000; 83: 1-19.

2. De Ponti F, Poluzzi E, Montanaro N. Organising evidence on QT prolongation and occurrence of Torsades de Pointes with non-antiarrhythmic drugs: A call for consensus. Eur J Clin Pharmacol 2001; 57: 185-209.

3. Sugiyama A, Satoh Y, Hashimoto K. Acute electropharmacological effects of intravenously administered amiodarone assessed in the in vivo canine model. Jpn J Pharmacol 2001; 87: 74-82.

4. Sugiyama A, Hashimoto K. Effects of gastrointestinal prokinetic agents, TKS159 and cisapride, on the in situ canine heart assessed by cardiohemodynamic and electrophysiological monitoring. Toxicol Appl Pharmacol 1998; 152: 261-269.

5. Sugiyama A, Aye NN, Katahira S, Saitoh M, Hagihara A, Matsubara $\mathrm{Y}$, et al. Effects of nonsedating antihistamine, astemizole, on the in situ canine heart assessed by cardiohemodynamic and monophasic action potential monitoring. Toxicol Appl Pharmacol 1997; 143: 8995.

6. Sugiyama A, Aye NN, Katahira S, Hagihara A, Matsubara Y, Hashimoto K. Effects of magnesium sulfate on the canine cardiovascular system complicating astemizole overdose. J Cardiovasc Pharmacol 1997; 29: 795-800.

7. Satoh Y, Sugiyama A, Tamura K, Hashimoto K. Effects of a class III antiarrhythmic drug, dofetilide, on the in situ canine heart assessed by the simultaneous monitoring of hemodynamic and electrophysiological parameters. Jpn J Pharmacol 1999; 81: 79-85.

8. Shiina H, Sugiyama A, Takahara A, Satoh Y, Hashimoto K. Comparison of the electropharmacological effects of verapamil and propranolol in the halothane-anesthetized in vivo canine model under monophasic action potential monitoring. Jpn Circ J 2000; 64: 777 782.

9. Sugiyama A, Satoh Y, Hashimoto K. In vivo canine model comparison of cardiohemodynamic and electrophysiological effects of a new antipsychotic drug aripiprazole (OPC-14597) to haloperidol. Toxicol Appl Pharmacol 2001; 173: 120-128.

10. Satoh Y, Sugiyama A, Chiba K, Tamura K, Hashimoto K. QTprolonging effects of sparfloxacin, a fluoroquinolone antibiotic, assessed in the in vivo canine model with monophasic action potential monitoring. J Cardiovasc Pharmacol 2000; 36: 510-515.

11. Satoh Y, Sugiyama A, Tamura K, Hashimoto K. Effects of magnesium sulfate on the haloperidol-induced QT prolongation assessed in the canine in vivo model under the monitoring of monophasic action potential. Jpn Circ J 2000; 64: 445-451.

12. Wharton JM, Hill JN, Freedberg NA, Prystowsky EN. Disopyramide. In: Messerli FH, editor. Cardiovascular drug therapy, 2nd ed. Philadelphia: Saunders; 1996: 1273-1295.

13. Podrid PJ. Mexiletine. In: Messerli FH, editor. Cardiovascular drug therapy, 2nd ed. Philadelphia: Saunders; 1996: 1319-1331.

14. Satoh Y, Sugiyama A, Tamura K, Hashimoto K. Effects of mexiletine on the canine cardiovascular system complicating cisapride 
overdose: Potential utility of mexiletine for the treatment of druginduced long QT syndrome. Jpn J Pharmacol 2000; 83: 327-334.

15. Franz MR. Bridging the gap between basic and clinical electrophysiology: What can be learned from monophasic action potential recordings? J Cardiovasc Electrophysiol 1994; 5: 699-710.

16. Franz MR, Costard A. Frequency-dependent effects of quinidine on the relationship between action potential duration and refractoriness in the canine heart in situ. Circulation 1988; 77: 1177-1184.

17. Kirchhof PF, Fabritz CL, Franz MR. Postrepolarization refractoriness versus conduction slowing caused by class I antiarrhythmic drugs: Antiarrhythmic and proarrhythmic effects. Circulation 1998; 97: $2567-2574$.

18. Bazett HC. An analysis of the time-relations of electrocardiogram. Heart 1920; 7: 353-370.

19. Sugiyama A, Hashimoto K. Effects of a typical IKr channel blocker sematilide on the relationship between ventricular repolarization, refractoriness and onset of torsades de pointes. Jpn J Pharmacol 2002; 88: 414-421.

20. Hashimoto K, Haruno A, Matsuzaki T, Sugiyama A, Akiyama, K. Effects of antiarrhythmic drugs on canine ventricular arrhythmia models: Which electrophysiological characteristics of drugs are related to their effectiveness? Cardiovasc Drugs Ther 1991; 5: $805-$ 818.

21. Sugiyama A, Motomura S, Tamura K, Hashimoto K. Comparison of cardiovascular effects of pirmenol with those of disopyramide in isolated canine heart preparations cross-circulated with a donor dog. Jpn J Pharmacol 1990; 53: 97-110.

22. Sugiyama A, Motomura S, Hashimoto K. Utilization of an isolated, blood-perfused canine papillary muscle preparation as a model to assess efficacy and adversity of class I antiarrhythmic drugs. Jpn J Pharmacol 1994; 66: 303-316.

23. Task Force of the Working Group on Arrhythmias of the European Society of Cardiology. The Sicilian gambit: A new approach to the classification of antiarrhythmic drugs based on their actions on arrhythmogenic mechanisms. Circulation 1991; 84: 1831-1851.

24. Yatani A, Akaike N. Blockage of the sodium current in isolated single cells from rat ventricle with mexiletine and disopyramide. J Mol Cell Cardiol 1985; 17: 467-476.

25. Coraboeuf E, Deroubaix E, Escande D, Coulombe A. Comparative effects of three class I antiarrhythmic drugs on plateau and pacemaker currents of sheep cardiac Purkinje fibers. Cardiovasc Res 1988; 22: 375-384.

26. Wu B, Sato T, Kiyosue T, Arita M. Blockade of 2,4-dinitrophenol induced ATP sensitive potassium current in guinea pig ventricular myocytes by class I antiarrhythmic drugs. Cardiovasc Res 1992; 26: 1095-1101.

27. Virág L, Varró A, Papp JG. Effect of disopyramide on potassium currents in rabbit ventricular myocytes. Naunyn-Schmiedeberg's Arch Pharmacol 1998; 357: 268-275.

28. Watanabe Y, Hara Y, Tamagawa M, Nakaya H. Pirmenol inhibits muscarinic acetylcholine receptor-operated $\mathrm{K}^{+}$current in the guinea pig heart. Eur J Pharmacol 1997; 338: 71-74.

29. Sadanaga T, Ogawa S, Okada Y, Tsutsumi N, Iwanaga S, Yoshikawa $\mathrm{T}$, et al. Clinical evaluation of the use-dependent QRS prolongation and the reverse use-dependent QT prolongation of class I and class III antiarrhythmic agents and their value in predicting efficacy. Am Heart J 1993; 126: 114-121.

30. Kiyosue T, Arita M. Late sodium current and its contribution to action potential configuration in guinea pig ventricular myocytes. Circ Res 1989; 64: 389-397.

31. Sato T, Shigematsu S, Arita M. Mexiletine-induced shortening of the action potential duration of ventricular muscles by activation of ATP-sensitive $\mathrm{K}^{+}$channels. Br J Pharmacol 1995; 115: 381-382.

32. Sugiyama A, Ishida Y, Satoh Y, Aoki S, Hori M, Akie Y, et al. Electrophysiological, anatomical and histological remodeling of the heart to AV block enhances susceptibility to arrhythmogenic effects of QT-prolonging drugs. Jpn J Pharmacol 2002; 88: 341-350. 